\title{
Phase diagram of the adhesive hard sphere fluid
}

\author{
Mark A. Miller \\ University Chemical Laboratory, Lensfield Road, Cambridge CB2 1EW, U. K. \\ Daan Frenkel \\ FOM Institute for Atomic and Molecular Physics, \\ Kruislaan 407, 1098 SJ Amsterdam, The Netherlands
}

(Dated: October 29, 2018)

\begin{abstract}
The phase behavior of the Baxter adhesive hard sphere fluid has been determined using specialized Monte Carlo simulations. We give a detailed account of the techniques used and present data for the fluid-fluid coexistence curve as well as parametrized fits for the supercritical equation of state and the percolation threshold. These properties are compared with the existing results of Percus-Yevick theory for this system.
\end{abstract}

\section{INTRODUCTION}

For a pure substance to be capable of exhibiting equilibrium between two fluid phases - a gas and a liquidthe interaction between its particles must include an attractive component. Possibly the simplest model to incorporate attraction is the square-well potential, where particles present each other with a hard core and a uniform region of favorable potential energy up to an abrupt distance threshold beyond which there is no interaction. The square-well model has two intrinsic length scales: the hard-core diameter and the width of the attractive well. The ratio of these lengths determines the phase behavior.

In 1968, Baxter introduced a model fluid containing attraction but having only one length scale, the hardcore diameter 1 In this model, the attraction takes the form of an adhesive interaction when particles are actually in contact, and therefore has an effectively infinitesimal range. Baxter produced an analytic equation of state for this "adhesive hard sphere" model using the Percus-Yevick framework and compressibility equation, showing that the model possesses a critical point below which gas-liquid equilibrium may be observed. A second Percus-Yevick equation of state - derived from the energy equation - soon followed, ${ }^{2}$ with a strikingly different critical point from the compressibility-equation prediction.

The Percus-Yevick framework also furnishes the cluster size distribution. $\frac{3}{-}$ The surface adhesion provides an unambiguous definition of clusters as connected sets of mutually touching particles. At sufficiently low density, the fluid consists only of monomers and finite clusters, but above a temperature-dependent density threshold, clusters tend to aggregate into amorphous macroscopic structures. The system then contains sample-spanning infinite clusters and is said to percolate. The percolation threshold, at which the divergence of the cluster size occurs, has been located within the Percus-Yevick approximation by Chiew and Glandt. 3

Baxter regarded the adhesive hard sphere model as a highly idealized manifestation of a general potential with a repulsive core and an attractive tail. However, it has since been suggested ${ }^{4}$ that it is quite a reasonable description of colloidal systems, where the particles interact on length scales much smaller than their own size. The gas and liquid are then more appropriately regarded as lowand high-density colloidal fluid phases. Percolation can be an observable phenomenon in colloidal systems, and may sometimes be detected directly, such as by a sudden increase in electrical conductivity across the sample.$^{\frac{5}{-}}$ The Baxter model is particularly appealing in the light of recent theoretica ${ }^{6}$ and experimental ${ }^{7}$ work that predicts and confirms the existence of a re-entrant glass-liquidglass transition in colloidal systems with short-range attractive forces. For very short-range attraction there is also a glass-glass transition between a caged "repulsive" glass and an energetic "attractive" glass.

A major obstacle in the application of the adhesive hard sphere model to the description of experimental data is that the model's phase behavior is only known through the approximate and conflicting theoretical results of Percus-Yevick theory. Computer simulations 8.9.10.11 have provided some information on percolation, but have not tackled the issue of phase coexistence. The purpose of the present contribution is to provide a better knowledge of the adhesive hard sphere phase diagram using computer simulation. We describe in detail the special Monte Carlo techniques employed (Section III) and supply numerical results for the fluidfluid coexistence curve, the percolation threshold, and the supercritical equation of state (Section [V]). We begin, in Section III with a definition of the model itself. It will be seen that this definition involves taking a limit that introduces a singularity into the potential energy function - an alarming prospect at first sight. The model in its strictest interpretation is indeed pathological, 12 but the problem is rather more subtle than it first appears, and in fact starts to set in before the full limit is taken. A proper discussion of this point is deferred to Section IIID, where the difficulties can be put into the context of the simulation methodology. 


\section{THE ADHESIVE HARD SPHERE MODEL}

The adhesive hard sphere model is defined by the pair potential function 1

$$
U(r) / k T= \begin{cases}\infty & 0 \leq r<\sigma \\ \ln [12 \tau(1-\sigma / d)] & \sigma \leq r<d \\ 0 & d \leq r\end{cases}
$$

in the limit $d \rightarrow \sigma$, where $k T$ is the thermal energy and $r$ the particle separation. Eq. (1) describes a square well with hard-core diameter $\sigma$, width $d-\sigma$, and depth controlled by the parameter $\tau$.

Equation (1) couples the depth of the well to its width in such a way that the total Boltzmann weight of bound configurations remains finite as the well width is reduced to zero:

$$
\begin{aligned}
\lim _{d \rightarrow \sigma} \int_{\sigma}^{d} e^{-U(r) / k T} 4 \pi r^{2} d r & =\lim _{d \rightarrow \sigma} \frac{d}{12 \tau} \frac{4 \pi}{3}\left(d^{2}+\sigma d+\sigma^{2}\right) \\
& =\frac{\sigma}{12 \tau} 4 \pi \sigma^{2}
\end{aligned}
$$

Hence, in the limit $d \rightarrow \sigma$, which corresponds to an infinitesimally narrow but infinitely deep well, one can write

$$
e^{-U(r) / k T}=\Theta(r-\sigma)+\frac{\sigma}{12 \tau} \delta(r-\sigma)
$$

The Heaviside step function, $\Theta$, accounts for the hardcore repulsion, while the Dirac $\delta$ represents the surface adhesion. Eq. (3) is our working definition of the Baxter model.

In the limit $\tau \rightarrow \infty$, Eq. (3) reduces to the hard sphere potential, so $\tau^{-1}$ measures the strength of adhesion. Alternatively, $\tau$ itself can be regarded as the effective temperature, since the true thermal energy $k T$ has been absorbed into the definition of the potential and does not appear explicitly in the right-hand side of Eq. (3). In the following, we shall refer to $\tau$ as the temperature.

The configuration space of an $N$-particle adhesive hard sphere system can be notionally divided into subspaces within each of which the number of binary contacts is constant. Let

$$
\Omega_{N M}=\frac{1}{N !}\left(\frac{\sigma}{12}\right)^{M} \int \delta_{M}\left(\mathbf{r}^{3 N}\right) \prod_{i<j}^{N} \Theta\left(r_{i j}-\sigma\right) d \mathbf{r}^{3 N}
$$

where $r_{i j}$ is the separation of particles $i$ and $j$. The integral extends over all particle coordinates, but the only configurations that make a non-zero contribution are those in which no particles overlap and the total number of binary contacts is $M$. The latter requirement is enforced by the function $\delta_{M}\left(\mathbf{r}^{N}\right)$. We point out that the presence of delta-functions in the Boltzmann factor of a pair of adhesive hard spheres should not be interpreted as a holonomic constraint on the Lagrangian of the system but rather as the limit of an increasingly stiff bond.
In fact, if the bonds between adhesive hard spheres acted as holonomic constraints, then the integrand in Eq. (4) would include a Jacobian as a result of the integration over the (constrained) momenta (see e.g. Ref. 13), not to mention the fact that the dimensionality of the phase space would in that case depend on the number of bonds. Although we will not need to manipulate an explicit form for $\delta_{M}\left(\mathbf{r}^{N}\right)$, it may be formally expressed as a sum of $M$ fold products of $\delta$ functions:

$$
\delta_{M}\left(\mathbf{r}^{3 N}\right)=\sum_{\left\{i_{1}, \ldots i_{2 M}\right\}}^{N} \delta\left(r_{i_{1}, i_{2}}-\sigma\right) \ldots \delta\left(r_{i_{2 M-1}, i_{2 M}}-\sigma\right),
$$

where $r_{i j}$ is the separation of particles $i$ and $j$. The sum is over all combinations of the $2 M$ indices that give distinct products of $\delta$ functions but neither generate constraints on a 'self-separation' (such as $r_{i i}$ ) nor duplicate a constraint on any given separation within a single term in the sum.

The configuration integral now takes the succinct form of Eq. (5), which emphasizes the role of $\Omega_{N M}$ as an effective density of states:

$$
\begin{aligned}
Z_{N}(\tau) & =\frac{1}{N !} \int \exp \left[-\sum_{i<j}^{N} U\left(r_{i j}\right) / k T\right] d \mathbf{r}^{3 N} \\
& =\sum_{M} \Omega_{N M} \tau^{-M}
\end{aligned}
$$

This notation will be useful when we consider histogram reweighting.

\section{SIMULATION}

\section{A. Canonical Monte Carlo}

The singularity in Eq. (3) means that the conventional Monte Carlo algorithm, in which the test particle undergoes random displacements, is not applicable. The probability of a trial move bringing two particles exactly into contact vanishes, and the energy change associated with breaking such a contact is formally infinite. Nevertheless, the integrals in Eq. (4) are finite (though see Section IIID) and there is an equilibrium between states with different total numbers of contacts, even if the time scales for establishing the equilibrium diverge as the limit of vanishing potential range is taken. ${ }^{12}$ In simulations of the adhesive hard sphere model it is therefore necessary to employ moves that explicitly make or break contacts.

For our canonical simulations we adopt the method pioneered by Seaton and Glandt,, 14 and refined by Kranendonk and Frenkel ${ }^{10}$ In a conventional Monte Carlo simulation the Boltzmann weights of configurations are compared, where a configuration means a specification of all particle coordinates. In contrast, simulations of the adhesive hard sphere model proceed by comparing the weights of different coordination states. Here we use 
the phrase coordination state to mean a specification of which particles are touching each other. In general, many configurations are compatible with a given coordination state. Since the Hamiltonian depends only on the number of contacts in the system, all configurations belonging to the same coordination state have the same statistical weight. With these concepts in mind, the key features of the algorithm may be outlined as follows.

1. A particle (the "test particle") is chosen at random.

2. A list is made of the possible coordination states of the test particle with other particles in the system, keeping the positions of the latter fixed. The list always contains one item in which the test particle makes no contacts at all, and $N-1$ items where it touches just one of the other particles in the system. It also enumerates pairs of particles that are sufficiently close for the test particle to touch both simultaneously, as well as triplets compatible with three simultaneous contacts to the test particle.

3. For each coordination state in the list, a transition probability is calculated.

4. A coordination state is chosen at random with weight in proportion to its transition probability.

5. A uniformly distributed random configuration belonging to the chosen coordination state is chosen.

6. If the configuration generates a hard-sphere overlap with another particle in the system, the move is rejected, otherwise it is accepted.

We now show how to calculate the transition probabilities in step 3 so that repeated application of this procedure generates the correct Boltzmann distribution of configurations. The total relative unnormalized weight of a coordination state of the test particle is given by the integral of the Boltzmann factor over all configurations belonging to that coordination state. For the coordination state involving no contacts with other particles, this weight is the free volume accessible to the test particle, which we label $t$ :

$$
W^{(0)}=\int_{V} \prod_{i \neq t}^{N} \Theta\left(r_{t i}-\sigma\right) d \mathbf{r}_{t},
$$

$V$ being the volume of the simulation box. For a coordination state involving contact of the test particle with one other particle, $a$, the test particle is constrained to the accessible part of the surface of $a$ :

$$
W^{(1)}=\frac{\sigma}{12 \tau} \int_{V} \delta\left(r_{t a}-\sigma\right) \prod_{i \neq t}^{N} \Theta\left(r_{t i}-\sigma\right) d \mathbf{r}_{t} .
$$

Similarly, for coordination states involving simultaneous contact with two particles ( $a$ and $b)$ or three particles $(a$, $b$ and $c$ ), we have

$$
W^{(2)}=\left(\frac{\sigma}{12 \tau}\right)^{2} \int_{V} \delta\left(r_{t a}-\sigma\right) \delta\left(r_{t b}-\sigma\right) \prod_{i \neq t}^{N} \Theta\left(r_{t i}-\sigma\right) d \mathbf{r}_{t}
$$

and

$$
\begin{array}{r}
W^{(3)}=\left(\frac{\sigma}{12 \tau}\right)^{3} \int_{V} \delta\left(r_{t a}-\sigma\right) \delta\left(r_{t b}-\sigma\right) \delta\left(r_{t c}-\sigma\right) \times \\
\prod_{i \neq t}^{N} \Theta\left(r_{t i}-\sigma\right) d \mathbf{r}_{t} .
\end{array}
$$

Moves that create or destroy more than three contacts simultaneously are not considered in the algorithm. A particle can, however, attain a coordination number greater than three through suitable combinations of moves where three or fewer contacts are created.

If Eqs. (6) to (9) could be evaluated conveniently, the canonical distribution could be generated directly by choosing a coordination state at random using these weights. The transition probability would be

$$
\alpha_{i j}=\frac{W_{j}}{\sum_{k=1}^{n} W_{k}},
$$

where the possible coordination states of the test particle have been numbered from 1 to $n$, and the weight $W_{j}$ of state $j$ has been evaluated using the appropriate formula from Eqs. (6) to (9). Note that $\alpha_{i j}$ does not depend on $i$, and that all moves would be accepted, since new configurations never generate overlaps and are already chosen with the desired probability distribution.

The product of step functions that appears in Eqs. (6) to (9) to preclude overlaps with the remaining particles in the system, makes evaluation of the weights very difficult. Following Seaton and Glandt,, 14 we consider instead the modified weights, in which the overlap is initially ignored. The weight of the coordination state where the test particle makes no contacts is now a free integral over the container volume:

$$
W^{\prime(0)}=\int_{V} d \mathbf{r}_{t}
$$

The weight for a one-contact coordination state is an integral over the complete sphere of radius $\sigma$ surrounding a particle:

$$
W^{\prime(1)}=\frac{\sigma}{12 \tau} \int_{V} \delta\left(r_{t a}-\sigma\right) d \mathbf{r}_{t} .
$$

For two simultaneous contacts we integrate around a circle of configurations:

$$
W^{\prime(2)}=\left(\frac{\sigma}{12 \tau}\right)^{2} \int_{V} \delta\left(r_{t a}-\sigma\right) \delta\left(r_{t b}-\sigma\right) d \mathbf{r}_{t}
$$

and for three contacts, only two configurations (located symmetrically above and below the plane defined by $a, b$ 
and c) contribute:

$$
W^{\prime(3)}=\left(\frac{\sigma}{12 \tau}\right)^{3} \int_{V} \delta\left(r_{t a}-\sigma\right) \delta\left(r_{t b}-\sigma\right) \delta\left(r_{t c}-\sigma\right) d \mathbf{r}_{t} .
$$

The integrals in Eqs. (12) to (14) can be evaluated by suitable series of variable changes. Alternatively, one may consider first a square-well potential of finite width. Coordination states with 1, 2 and 3 contacts then restrict the test particle's center-of-mass to, respectively, a spherical shell, a torus with diamond-shaped cross-section, and a pair of parallelepipeds. Taking the limit of infinitesimal well width then produces the same results as direct integration of the delta functions. One obtains

$$
\begin{aligned}
W^{\prime(0)} & =V, \\
W^{\prime(1)} & =\frac{\pi \sigma^{3}}{3 \tau}, \\
W^{\prime(2)} & =\frac{\pi \sigma^{4}}{72 \tau^{2} r_{a b}}, \\
W^{\prime(3)} & =\left(\frac{\sigma}{12 \tau}\right)^{3} \frac{2 r_{t a} r_{t b} r_{t c}}{\left|\left(\mathbf{r}_{t a} \times \mathbf{r}_{t b}\right) \cdot \mathbf{r}_{t c}\right|} .
\end{aligned}
$$

Unlike previous workers,, 9.15 we consider moves to all triplets of particles capable of simultaneously touching the test particle, not just to those triplets where $a, b$ and $c$ are all mutually touching. The coordination states neglected by the latter approach can be reached by combinations of other moves, but including the direct moves should make the algorithm more efficient at low temperature or high density, where the average coordination number is expected to be high.

It is the weights in Eqs. (15) to (18) that we use for the transition probability in step 4 of the Monte Carlo algorithm:

$$
\alpha_{i j}^{\prime}=\frac{W_{j}^{\prime}}{\sum_{k=1}^{n} W_{k}^{\prime}} .
$$

To show that this choice satisfies detailed balance and leads to the correct Boltzmann distribution, let $f_{j}=$ $W_{j} / W_{j}^{\prime}$ be the fraction of configurations belonging to coordination state $j$ that are not blocked by overlap with other particles. The rejection of overlaps in step 6 of the algorithm means that a trial move to coordination state $j$ will be accepted with probability $p_{i j}^{\text {acc }}=f_{j}$. The overall probability of moving to state $j$ from state $i$ is therefore

$$
\pi_{i j}=\alpha_{i j}^{\prime} p_{i j}^{\mathrm{acc}}=\frac{W_{j}}{\sum_{k=1}^{n} W_{k} / f_{k}} .
$$

Note that the sum in the denominator of Eq. (20) depends on the positions of all the particles except the test particle. Since states $i$ and $j$ differ only by the position of the test particle, this sum is the same for the reverse move, so that $W_{i} \pi_{i j}=W_{j} \pi_{j i}$, and detailed balance is satisfied. Recalling that we do not attempt moves that create more than three contacts simultaneously, detailed balance also requires that an attempted displacement of any particle that has accumulated more than three contacts is automatically rejected.

Having chosen a coordination state in step 4 of the algorithm, step 5 requires a uniformly distributed random configuration from this state to be generated. For coordination states involving $0,1,2$ or 3 contacts one must therefore select, respectively, a random point in the simulation box, a random point on a sphere surrounding particle $a$, a random point on the circle of contact with particles $a$ and $b$, or one of the two points lying a distance $\sigma$ from each of $a, b$ and $c$.

In practice, it is too time-consuming to enumerate all possible coordination states of the test particle, and this task scales unfavorably with the system size. It is therefore desirable to restrict movement of the test particle to the vicinity of its original position, so that only a subset of the possible coordination states need be identified and considered. However, as discussed by Seaton and Glandt, $\stackrel{14}{=}$ care must be taken since the normalizing sum in Eq. (20) then depends on the original position of the particle. Kranendonk and Frenke ${ }^{10}$ have prescribed a solution to the problem: a random point is chosen within a sphere of radius $r_{\text {test }}$ centered on the original position of the test particle, and this point is used as the center of a second sphere (the test sphere), also of radius $r_{\text {test }}$. Displacements of the test particle are then considered to coordination states within the test sphere. The chance of choosing the same test sphere in the reverse move is identical, so that the modified scheme maintains detailed balance.

The test sphere introduces another complication in that it may intersect some of the spherical and circular contact surfaces available to the test particle, thereby excluding a fraction of the configurations belonging to the corresponding coordination states. One must therefore calculate what fraction of each contact surface lies within the test sphere, and modify the weights in Eq. (19) by these fractions when calculating the transition probabilities in step 3 of the algorithm. In step [5 one must then choose a uniformly distributed random configuration restricted to the part of the surface that lies within the test sphere.

\section{B. Grand Canonical Monte Carlo}

Since two phases can only coexist when their pressures are equal, computational studies of coexistence often employ an ensemble where the size of the simulation cell can fluctuate in response to the pressure. The coordinates of the particles or the cluster centers-of-mass are typically scaled in proportion to the change in the cell length. In the adhesive hard sphere fluid, clusters can percolate even at moderately low density. A percolating cluster spans the simulation cell, so that increasing or decreasing the cell size in the presence of such a cluster would always involve breaking a contact or generating an overlap be- 
tween at least two particles. Since overlaps are forbidden, and the breaking of a bond is energetically prohibited, all trial changes in the cell size would be rejected. Isobaricisothermal and Gibbs-ensemble simulations therefore fail as soon as the percolation threshold is crossed.

To study density fluctuations, we work instead in the grand canonical ensemble, where the volume of the simulation is fixed, but the number of particles fluctuates in response to the imposed chemical potential. To avoid the formally infinite change in potential energy associated with the creation and destruction of contacts between particles, we only consider the insertion and removal of particles whose coordination number is zero. The procedure is therefore identical to that used for ordinary hard spheres, $\stackrel{16}{=}$ with the slight modification that a removal must be rejected if the chosen particle happens to have a non-zero coordination number. In our grand canonical simulations, particle insertion and removal steps are attempted with equal probability totaling $45 \%$.

In an attempt to accelerate equilibration, we also perform cluster translation moves with probability $5 \%$. A particle is chosen at random, and then all particles in the cluster to which it belongs are translated by the same amount. The maximum translation in each Cartesian direction is inversely proportional to the number of particles in the cluster.

\section{Parallel Tempering}

At low temperature and high density, the formation of large, highly-coordinated clusters drastically slows down the equilibration of the simulations. To help overcome this problem, we employ the parallel tempering scheme of Geyer ${ }^{17}$ in the grand canonical ensemble. In our implementation, several runs are performed simultaneously at the same temperature and a series of increasing chemical potentials. At sufficiently low chemical potential, one can always recover an ergodic dilute gas. Parallel tempering involves periodic attempts to exchange the configurations of pairs of adjacent runs in the hierarchy of chemical potentials. The acceptance probability of such moves is given below and ensures that the correct grand canonical distribution is obtained for each run individually. The advantage of swapping configurations is that a large cluster formed at high chemical potential breaks down when transferred to lower chemical potential, while a new cluster is built up from the configuration received by the run at higher chemical potential.

Using Eq. (5), we can write the grand partition function of the adhesive hard sphere model as

$$
\Xi(\tau, z)=\sum_{N=0}^{\infty}\left(\frac{e^{\mu / k T}}{\Lambda^{3}}\right)^{N} Z_{N}(\tau)=\sum_{N, M} \Omega_{N M} z^{N} \tau^{-M},
$$

where the chemical potential $\mu$ enters through the activity $z=\Lambda^{-3} \exp (\mu / k T)$ and $\Lambda$ is the thermal de Broglie wavelength. The probability of observing the system in a configuration with $N$ particles and $M$ contacts under conditions of effective temperature $\tau$ and activity $z$ is

$$
P_{N M}(\tau, z)=\Omega_{N M} z^{N} \tau^{-M} / \Xi(\tau, z) .
$$

The joint probability of a system having $N_{1}$ particles and $M_{1}$ contacts at temperature $\tau$ and activity $z_{1}$ at the same time as a second system with equal temperature but activity $z_{2}$ has $N_{2}$ particles and $M_{2}$ contacts is simply the product $P=P_{N_{1} M_{1}}\left(\tau, z_{1}\right) P_{N_{2} M_{2}}\left(\tau, z_{2}\right)$. The equilibrium probability of observing a pair of systems under the same conditions but with the configurations exchanged is $P^{\prime}=P_{N_{2} M_{2}}\left(\tau, z_{1}\right) P_{N_{1} M_{1}}\left(\tau, z_{2}\right)$. Since the probability of attempting a configuration exchange is independent of configuration, the correct joint distribution is sampled if exchanges are accepted with probability

$$
p_{\mathrm{ex}}^{\mathrm{acc}}=\min \left[1, P^{\prime} / P\right]=\min \left[1,\left(z_{1} / z_{2}\right)^{N_{2}-N_{1}}\right] .
$$

The activities must be chosen close enough that typical values of $p_{\mathrm{ex}}^{\mathrm{acc}}$ are not too small. As the extensivity of the exponent in Eq. (23) makes clear, $p_{\mathrm{ex}}^{\text {acc }}$ decreases with increasing system size because of the decreasing relative size of the fluctuations. It is therefore necessary to use a larger number of parallel runs with more closely spaced activities for larger systems.

A natural companion of the parallel tempering technique is multiple histogram reweighting, ${ }^{18,19}$ which allows the results from simulations of different conditions to be combined. One may then obtain rigorously interpolated data for conditions different from those explicitly simulated without the need for further runs. Our implementation of this method is detailed in the Appendix.

\section{Inherent Pathology}

It has been pointed out that for systems of $N \geq 12$ adhesive hard spheres, the configuration integral $Z_{N}$ diverges $\stackrel{12.20}{=}$ and that the model is therefore pathological. The problem arises from certain maximally connected clusters in which the number of contacts between particles exceeds the number of vibrational degrees of freedom, leading to non-integrable singularities in $Z_{N}$.

The present simulations are concerned with the fluid phases of Baxter's model. Under conditions where dense clusters form, aggregation tends to proceed in a polytetrahedral manner, with particles attaching to existing triangular facets due to the contact adhesion. The clusters that lead to the divergence of $Z_{N}$ are close-packed and characteristic of the crystal phase. In contrast, regular tetrahedra are not space-filling, and so troublesome clusters are increasingly unlikely to arise spontaneously in the simulation as $\tau$ decreases. Indeed, for such a shortrange potential, the liquid phase is expected to be highly metastable with respect to the crystal, ${ }^{21}$ with a high free energy barrier between the two. Here we effectively neglect the diverging contributions of crystal nuclei containing 12 or more particles. 
In the event that a pathological cluster were to arise in the simulation, the algorithm described in this section would simply fail to observe the additional contact between particles that leads to non-integrability. Since such "accidental" contacts arise from the geometrical packing of strictly monodisperse spheres, the algorithm effectively introduces an infinitesimal polydispersity. For implications regarding the applicability of the model, we refer readers to the detailed discussion by Stell 12

\section{RESULTS}

\section{A. Coexistence Curve}

A grand canonical simulation yields a histogram of the number of particles in the system-effectively the probability distribution of the reduced particle density, $\rho=\sigma^{3} N / L^{3}$, where $L$ is the length of the cubic simulation box. We work throughout with $\rho$, which is related to the volume fraction $\eta$ of the spheres by $\eta=\rho \pi / 6$.

At any subcritical temperature, there is an activity at which the probability distribution of $\rho$ is bimodal with peaks of equal area, signifying equilibrium between a lowand a high-density fluid phase. The coexisting densities are given by the mean of each peak. In practice, the finite size of the simulation box means that for modestly subcritical temperatures, the two peaks overlap, and it becomes impossible to attribute the intermediate densities to one peak or the other. It is therefore more reliable to define equilibrium by equal heights of the peaks, and the coexisting densities by the peak positions.

We note that, at significantly subcritical temperatures, where two separated peaks are observed, the effect of small changes in the activity about the coexistence point is to alter the relative heights of the peaks without affecting much their positions. Under these conditions, the positions of the peaks at coexistence are also not very sensitive to the system size. Closer to the critical point, discrepancies due to the definition of coexistence and finite size effects both increase. However, using the peak heights is far less ambiguous than using their areas.

Table I reports the coexisting (peak) densities as a function of the temperature parameter $\tau$ in a cubic simulation box of length $L=8 \sigma$. Also given are an estimate of the statistical error and a measure of the size dependence of the results by comparison with simulations at $L=6 \sigma$. At the lowest temperatures, where the coexisting density peaks are well separated, the difference between the peak positions and their integrated means is roughly 0.01 , the direction of the differences being such that the means of the two peaks are closer than their peak positions.

The pronounced size dependence near the top of the low- and high-density branches was noted in previous work, ${ }^{22}$ and was exploited to derive the critical point in the thermodynamic limit, as listed in the last line of Table III At this point, the adhesive hard sphere density distribution can be scaled onto the well-known ${ }^{23}$ uni-
TABLE I: Coexisting peak densities $\rho_{\mathrm{lo}}$ and $\rho_{\mathrm{hi}}$ of the lowand high-density fluid phases of the adhesive hard sphere model with simulation cell length $L=8 \sigma . \delta_{\text {ran }}$ denotes the uncertainty in locating the peak positions. $\delta_{\text {sys }}$ is the average of the decrease in $\rho_{\mathrm{lo}}$ and the increase in $\rho_{\mathrm{hi}}$ when the simulation cell size is decreased to $L=6 \sigma$. This quantity is an indication of the increasing size-dependence of the results as the critical point is approached from below.

\begin{tabular}{lllll}
\hline \hline$\tau$ & $\rho_{\text {lo }}$ & $\rho_{\text {hi }}$ & $\delta_{\text {ran }}$ & $\delta_{\text {sys }}$ \\
\hline 0.090 & 0.076 & 0.860 & 0.01 & 0.005 \\
0.092 & 0.084 & 0.857 & 0.01 & 0.005 \\
0.094 & 0.092 & 0.848 & 0.01 & 0.005 \\
0.096 & 0.102 & 0.842 & 0.01 & 0.005 \\
0.098 & 0.113 & 0.840 & 0.01 & 0.005 \\
0.100 & 0.125 & 0.830 & 0.01 & 0.005 \\
0.102 & 0.137 & 0.826 & 0.005 & 0.005 \\
0.104 & 0.152 & 0.818 & 0.005 & 0.01 \\
0.106 & 0.170 & 0.807 & 0.005 & 0.015 \\
0.108 & 0.192 & 0.795 & 0.005 & 0.02 \\
0.110 & 0.221 & 0.783 & 0.01 & 0.03 \\
0.112 & 0.256 & 0.752 & 0.01 & 0.03 \\
0.113 & 0.279 & 0.736 & 0.01 & 0.04 \\
0.114 & 0.31 & 0.72 & 0.03 & 0.06 \\
0.115 & 0.34 & 0.67 & 0.04 & 0.1 \\
\hline \hline
\end{tabular}

TABLE II: Critical temperature and density of the adhesive hard sphere model from the Percus-Yevick compressibility ${ }^{1}$ and energy ${ }^{2}$ routes, and from simulation. ${ }^{22}$

\begin{tabular}{lll}
\hline \hline method & $\tau_{c}$ & $\rho_{c}$ \\
\hline PY compressibility & 0.0976 & 0.232 \\
PY energy & 0.1185 & 0.609 \\
simulation & $0.1133 \pm 0.0005$ & $0.508 \pm 0.01$ \\
\hline \hline
\end{tabular}

versal critical order-parameter distribution of the threedimensional Ising model at any system size $24,25.26 \quad(5 \leq$ $L / \sigma \leq 10$ were studied). However, it is clear from Table II and Fig. 1] that the finite-system coexistence branches significantly overshoot the critical point. It is partly because of the varying finite-size effects that we have refrained from fitting the coexistence curve to a functional form, such as a scaling law with universal exponents. We also point out that the parameter $\tau$ is not the thermodynamic temperature in the usual sense. Even if temperature is the relevant parameter in an experimental realization of adhesive hard spheres, its relation to $\tau$ depends entirely on the substance, and could be linear, 27 inverse, 28 or more complicated, 5

Table III and Fig. 11 emphasize the large discrepancies in the critical point and coexistence curve derived from the compressibility ${ }^{1}$ and energy $^{2}$ routes of Percus-Yevick theory; the critical densities differ by almost a factor of three. The present simulations indicate that the true properties lie between the two theoretical results, but somewhat closer to the energy route, despite the fact that the compressibility results are more often used when modeling experimental data. 


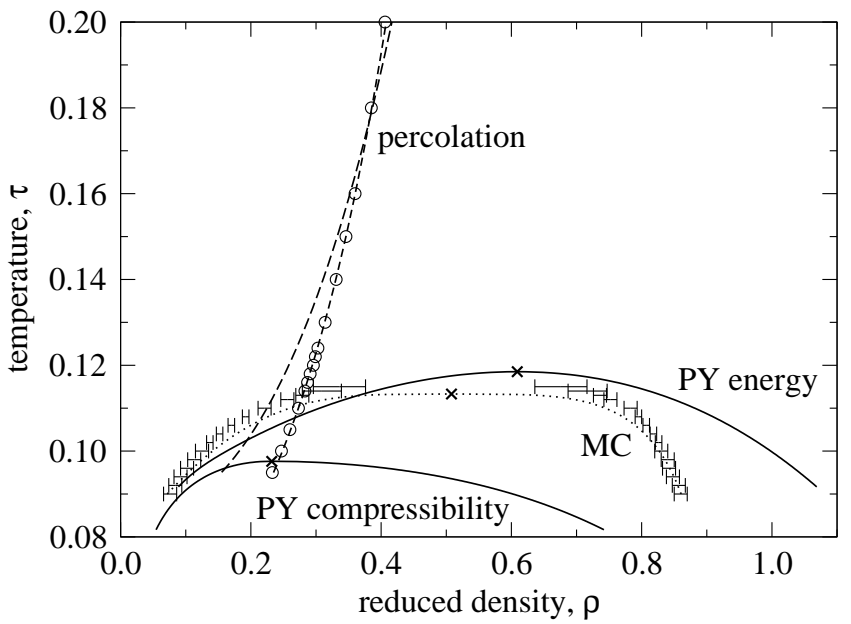

FIG. 1: Phase diagram of the adhesive hard sphere fluid. The two solid lines are the fluid-fluid coexistence curves from Percus-Yevick theory as marked. The simulation coexistence data from Table \are shown by points with error bars (marking statistical uncertainty), with a dotted line merely to guide the eye. Critical points are indicated by crosses. The dashed lines show the percolation threshold: long dashes are the Percus-Yevick result, Eq. (25) and short dashes with circles are the simulation results and their fit, Eq. 24). To the highdensity side of the threshold the system essentially always contains an infinite cluster.

\section{B. Percolation Threshold}

In principle, a percolating cluster is a dynamical object. A system-spanning cluster may assemble, but its constituent particles are in dynamic equilibrium with the rest of the system. In a simulation, one can therefore define a percolation probability equal to the fraction of the encountered configurations that contain a systemspanning cluster. At a given temperature, this probability is a sigmoidal function of density, rising from zero to unity in a relatively narrow range. The density at which the probability passes through 0.5 has been found to be quite insensitive to the system size $e^{10.11}$ and we therefore adopt this point as a robust definition of the percolation density, $\rho_{\text {perc }}(\tau)$. For $\rho>\rho_{\text {perc }}$ the system effectively always contains a percolating cluster.

We have measured $\rho_{\text {perc }}(\tau)$ in canonical simulations of a system with $N=500$ particles. The decreasing density at which percolation sets in as $\tau$ is lowered means that the ergodicity problems associated with dense lowtemperature regime do not arise, and parallel tempering is not necessary. Our data are well reproduced by a ratio of polynomials with six adjustable parameters:

$$
\rho_{\text {perc }}(\tau)=\frac{-10.09+182.4 \tau+606.9 \tau^{2}+15.31 \tau^{3}}{1+507.9 \tau+548.9 \tau^{2}},
$$

applicable in the range $0.095 \leq \tau \leq 3$. The maximum residuals between the measured and fitted densities are 0.001 for the portion above $\tau=0.2$ and 0.002 below it. These errors are equal to the estimated uncertainty

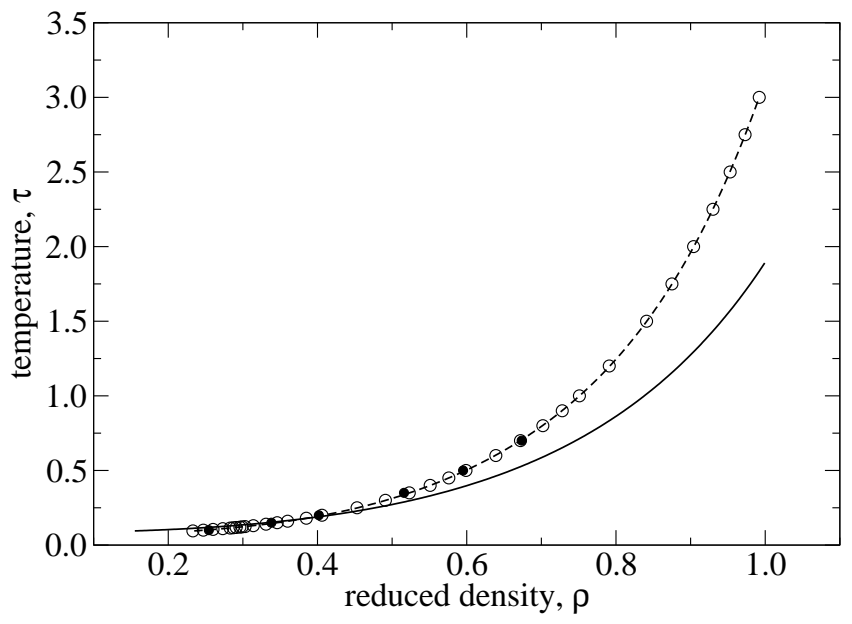

FIG. 2: Percolation threshold of the adhesive hard sphere fluid. Open circles are Monte Carlo data from this work, and the dashed line is the fit Eq. (24). Filled circles are Monte Carlo data from Table I of Ref. 11. The solid line is the Percus-Yevick result.

in the simulation data, as ascertained by independent simulations with different random number seeds.

Finite-size effects are relatively small in the percolation curve. Reducing the system size to $N=256$ particles shifts the curve systematically to higher density by about 0.001 towards the high- $\tau$ end of the range studied and 0.003 at the low- $\tau$ end.

Figure 2 shows the simulation data and the fit in Eq. (24) over the full range studied. We note that, at high $\tau$, the threshold density enters the two-phase crystal-fluid region of ordinary hard spheres $(0.939<$ $\rho<1.038) 29$ The onset of hard sphere behavior places an upper limit on the value of $\tau$ to which we can trace the percolation threshold. No meaning should be attached to the empirical fit in Eq. (24) outside the specified range.

Lee ${ }^{11}$ has performed a detailed scaling analysis of percolation in adhesive hard spheres in the range $0.1 \leq \tau \leq$ 0.7, and Fig. 2] shows that the latter results are consistent with the present work. Also plotted is the Percus-Yevick percolation threshold derived by Chiew and Glandt from the divergence of the mean cluster size with temperature at a given density: ${ }^{3}$

$$
\tau_{\text {perc }}(\eta)=\frac{19 \eta^{2}-2 \eta+1}{12(1-\eta)^{2}}, \quad \eta=\rho \pi / 6
$$

This formula increasingly overestimates the percolation density at given temperature for $\tau>0.177$, but underestimates it at lower temperatures approaching the twophase regime (Fig. 1).

Importantly, Fig. (1) shows that the critical point for the fluid-fluid phase transition lies well within the percolated regime, in contrast to the results of the PercusYevick compressibility results that suggest the critical point is rather close to the threshold. In an experimental system with very short range attractive forces, the 
onset of infinite clusters might therefore interfere with equilibrium phase separation and critical behavior ${ }^{30}$

\section{Equation of State}

In the grand canonical ensemble, the pressure can be derived from the average density $\langle\rho(z, \tau)\rangle$ as a function of the activity $z$ and temperature $\tau$. For a change in chemical potential at constant volume and temperature, $V d P=\langle N\rangle d \mu$. Integration yields an isotherm of the reduced pressure $P^{*}=\sigma^{3} P / k T$ as a function of the reduced density:

$$
P^{*}(\rho, \tau)=\int_{z=0}\langle\rho(z, \tau)\rangle d(\ln z)
$$

Below the critical point, one must integrate the mean densities of the two fluid phases separately, determining the constant of integration for the high-density branch by the requirement that the pressures be equal at the coexistence activity.

The multiple histogram technique is invaluable in evaluating Eq. (26), since it allows the density to be found effectively as a continuous function of the activity from a series of simulations at discrete points. Equally, one can interpolate between simulations at two values of $\tau$ to obtain an intermediate isotherm.

We have performed grand canonical parallel tempering simulations spanning a wide range of particle densities $(0<\rho<1)$ at each of $\tau=0.1,0.105,0.11,0.12,0.13$, $0.15,0.2,0.25,0.35,0.5,1,1.5,2,3$, and 5 using a simulation box of length $L=8 \sigma$. These directly-measured isotherms were supplemented by 22 intermediate temperatures derived by histogram reweighting from the pair of simulations bracketing each temperature.

The rather unconventional definition of the adhesive hard sphere model in Eq. (11) has consequences for fitting of an empirical equation of state to the data $P(\rho, \tau)$. Firstly, the high temperature limit is not an ideal gas but a system of hard spheres, whose equation of state is reproduced accurately by Speedy's formula: $:^{31}$

$$
P_{\mathrm{HS}}^{*}=\rho\left[1+\frac{x+0.076014 x^{2}+0.019480 x^{3}}{1-0.548986 x+0.075647 x^{2}}\right]
$$

where $x=B_{2}^{\mathrm{HS}} \rho$ and $B_{2}^{\mathrm{HS}}=2 \pi / 3$ is the reduced second virial coefficient of hard spheres. Additionally, the first four virial coefficients of adhesive hard spheres are known analytically ${ }^{32}$ consisting of the corresponding temperature-independent hard-sphere part plus a contribution from the adhesion. The adhesive contributions are

$$
\begin{aligned}
B_{2}^{\mathrm{adh}}(\tau)= & -\frac{B_{2}^{\mathrm{HS}}}{4 \tau} \\
B_{3}^{\mathrm{adh}}(\tau)= & \left(\frac{B_{2}^{\mathrm{HS}}}{4}\right)^{2}\left(-5 \tau^{-1}+\tau^{-2}-\frac{1}{18} \tau^{-3}\right), \\
B_{4}^{\mathrm{adh}}(\tau)= & \left(\frac{B_{2}^{\mathrm{HS}}}{4}\right)^{3}\left(-13.77358 \tau^{-1}+6.114 \tau^{-2}\right. \\
& -1.518 \tau^{-3}+0.17398 \tau^{-4} \\
& -6.33514 \times 10^{-3} \tau^{-5}-6.51271 \times 10^{-5} \tau(30)
\end{aligned}
$$

With this information, it is already clear that many existing empirical equations of state are not of an appropriate form for this model. Even the popular modified Benedict-Webb-Rubin (mBWR) equation, ${ }^{33}$ which employs 33 adjustable parameters and has been successfully fitted to the Lennard-Jones fluid equation of state, ${ }^{34}$ is unsatisfactory as it stands: the terms containing fractional powers of temperature are redundant in the light of Eqs. (28) and (29), and its high temperature limit is not flexible enough to reproduce Eq. (27).

We have attempted to adapt the mBWR equation by removing unnecessary terms and inserting ones more appropriate to the adhesive hard sphere model. However, we have been unable to obtain a satisfactory fit across the full gamut of our simulation data and must therefore be satisfied with an empirical fit to the supercritical part of the equation of state, which we now present. We note that the supercritical regime is relevant to many experimental applications of the adhesive hard sphere model.

To guarantee the correct low-density (up to third order in $\rho$ ) and high-temperature limits, we write the full adhesive hard sphere pressure as

$$
P^{*}(\rho, \tau)=P_{\mathrm{HS}}^{*}(\rho)+B_{2}^{\mathrm{adh}}(\tau) \rho^{2}+B_{3}^{\operatorname{adh}}(\tau) \rho^{3}+P_{\mathrm{fit}}^{*}(\rho, \tau),
$$

and fit the final term to a ratio of mixed polynomials in $\rho$ and $1 / \tau$ :

$$
P_{\text {fit }}^{*}(\rho, \tau)=\frac{\sum_{\left\{a_{i j}\right\}} a_{i j} \rho^{i} / \tau^{j}}{1+\sum_{\left\{b_{i j}\right\}} b_{i j} \rho^{i} / \tau^{j}} .
$$

In Eq. (32) the sums are over the sets of terms, with coefficients $\left\{a_{i j}\right\}$ and $\left\{b_{i j}\right\}$, that have been chosen to provide the flexibility to reproduce the data.

We have used a standard nonlinear least-squares routine $e^{35}$ to optimize Eq. (32) to reproduce our simulated and interpolated isotherms $P^{*}(\rho, \tau)$ for the $L=8 \sigma$ system at 35 values of $\tau \geq 0.117$ (the apparent finite-system critical point lying just below $\tau=0.117$ ). Starting from a modest number of terms, contributions were inserted and removed according to their ability to improve the fit, and the final result was a set of 35 terms, 24 in the numerator and 11 in the denominator, as listed in Tables III and IV We emphasize that the fit is meaningless below the lowest temperature used in the parametrization, $\tau=0.117$, but may be applied at arbitrarily high $\tau$ since 
TABLE III: Numerator coefficients for the equation of state, Eq. (32).

\begin{tabular}{cccccc}
\hline \hline$i$ & $j$ & $a_{i j}$ & $i$ & $j$ & $a_{i j}$ \\
\hline 4 & 2 & -3.787821 & 6 & 4 & -0.1954175 \\
4 & 4 & -0.3184060 & 6 & 5 & -0.3832949 \\
4 & 5 & 0.1460575 & 7 & 1 & -207.9268 \\
4 & 6 & -0.01199764 & 7 & 2 & 301.5938 \\
5 & 1 & -24.12376 & 7 & 3 & -88.02139 \\
5 & 2 & 0.6172855 & 7 & 4 & 6.609781 \\
5 & 4 & -1.679166 & 8 & 1 & 114.6900 \\
5 & 5 & 0.05973859 & 8 & 2 & -155.9904 \\
5 & 6 & 0.01606935 & 8 & 3 & 42.26076 \\
6 & 1 & 111.7043 & 8 & 4 & -4.117703 \\
6 & 2 & -137.3363 & 8 & 5 & 0.1512075 \\
6 & 3 & 43.98811 & 8 & 6 & -0.003129547 \\
\hline \hline
\end{tabular}

TABLE IV: Denominator coefficients for the equation of state, Eq. (32).

\begin{tabular}{cccccc}
\hline \hline$i$ & $j$ & $b_{i j}$ & $i$ & $j$ & $b_{i j}$ \\
\hline 1 & 2 & 29.25140 & 3 & 2 & 85.86019 \\
1 & 3 & -3.259845 & 3 & 3 & -8.978804 \\
2 & 1 & 32.11475 & 4 & 1 & 24.33100 \\
2 & 2 & -88.09174 & 4 & 2 & -26.90679 \\
2 & 3 & 9.484818 & 4 & 3 & 2.751372 \\
3 & 1 & -57.07652 & & & \\
\hline \hline
\end{tabular}

Eq. (31) reverts smoothly to the ordinary hard sphere equation of state at $\tau=\infty$.

Despite the inapplicability of the fit below $\tau=0.117$, one can always obtain the pressure in the low density limit using the hard-sphere equation of state plus the exact adhesive contributions up to fourth order in $\rho$ as listed in Eqs. (28) - (30):

$$
P^{*}(\rho, \tau)=P_{\mathrm{HS}}^{*}(\rho)+B_{2}^{\operatorname{adh}}(\tau) \rho^{2}+B_{3}^{\operatorname{adh}}(\tau) \rho^{3}+B_{4}^{\operatorname{adh}}(\tau) \rho^{4} .
$$

In fact, the low-density side of the coexistence curve is sufficiently rarefied that Eq. (33) is quite satisfactory for the full gas-like branch of subcritical isotherms, except for $\tau$ very close to the critical point.

Returning to the supercritical equation of state, Fig. B shows the pressure along the $\tau=0.12$ isotherm to demonstrate the performance of the fit. Note that the simulation results can be shown as a continuous line, since the multiple histogram technique allows us to calculate the pressure on an arbitrarily fine grid. The figure also shows the (dimensionless) derivative $\left(\partial P^{*} / \partial \rho\right)_{\tau}$, which is related to the dimensionless isothermal compressibility $\kappa^{*}$ by $1 / \kappa^{*}=\rho\left(\partial P^{*} / \partial \rho\right)_{\tau}$. The fit reproduces the derivative acceptably, despite the derivative not being used in the fitting process. In fact, Fig. 3 is a somewhat pessimistic case, since the agreement between the simulation data and fit, especially for the derivative, improves with increasing $\tau$.

The actual (not fractional) difference between the simulation and the fit is plotted for three temperatures in

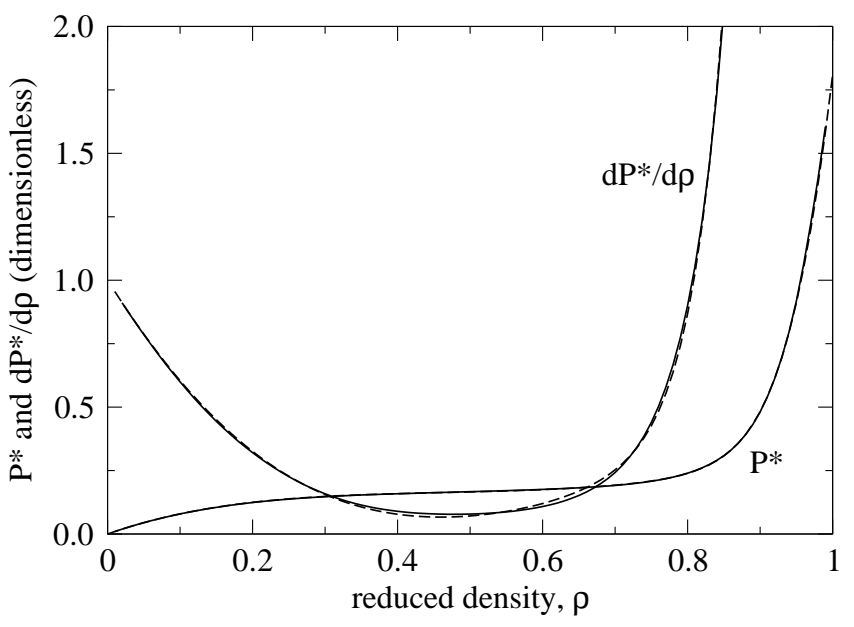

FIG. 3: Comparison of the $\tau=0.12$ isotherm from simulation and as reproduced by Eqs. (31) and (32). Also shown is the dimensionless derivative $\left(\partial P^{*} / \partial \rho\right)_{\tau}$ from both simulation and fit. In each case, the solid line is the simulation data and the dashed line the fit, though the two are not everywhere discernible.

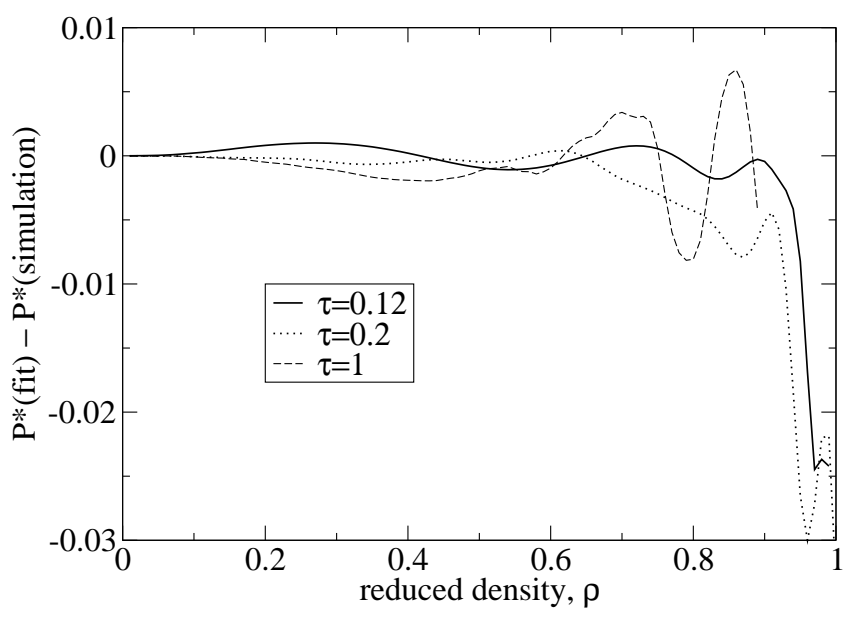

FIG. 4: Discrepancy (unscaled differences) between the pressure directly from simulations and the empirical form Eq. (32) for three temperatures.

Fig. 4. By construction, the discrepancy goes smoothly to zero at zero density. The increasing absolute size of the discrepancy at high densities still represents a small fractional error in the light of the steeply rising pressure in that regime (see Fig. 3). Approaching the critical point, the simulation data and fit suffer from finite-size effects. However, simulations with a smaller simulation cell of $L=6 \sigma$ produce almost indistinguishable results for $\tau \geq 0.14$, so the equation of state provided here is robust over a wide range of temperatures.

We now compare the simulation isotherms with the predictions of Percus-Yevick theory. Expressions for the pressure derived from the compressibility and energy routes, originally due to Baxter ${ }^{1}$ and Watts et al.,$\frac{2}{2}$ are given in convenient and explicit form by Barboy ${ }^{36}$ and 


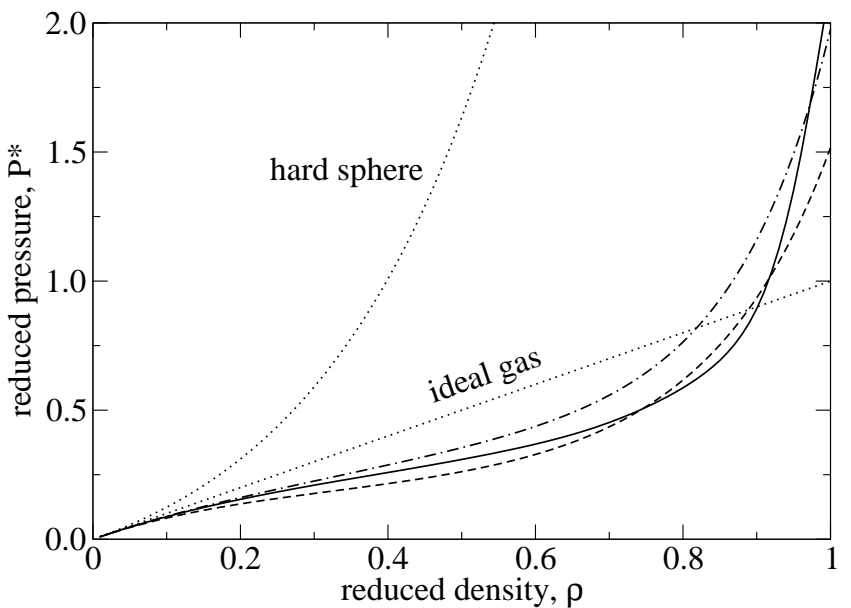

FIG. 5: Adhesive hard sphere pressure on a supercritical isotherm. The solid curve is the simulation result at $\tau=0.15$, equivalent to $\tau / \tau_{c}=1.3239$. The dashed and dot-dashed lines are the Percus-Yevick compressibility and energy results, respectively, each calculated at the same $\tau / \tau_{c}$ using the associated $\tau_{c}$. Shown by dotted lines are the ideal gas and ordinary hard sphere equations of state.

Tenne 37 Figure 5 shows the pressure along a supercritical isotherm at $\tau / \tau_{c}=1.3239$, which corresponds to $\tau=0.15$ using the critical temperature from simulations. The two Percus-Yevick results are plotted for the same value of the ratio $\tau / \tau_{c}$, but using their respective critical temperatures (Table II). Also plotted for comparison are the ideal gas equation of state and Eq. (27) for ordinary hard spheres. We see that the attractive component of the adhesive hard sphere potential can dramatically reduce the pressure with respect to hard spheres even at high density. Indeed, the attraction at this temperature is strong enough to overpower the effects of excluded volume to the extent that the adhesive hard sphere pressure lies below that of the ideal gas up to $\rho \approx 0.9$ (simulation data).

Figure [6 compares subcritical isotherms at $\tau / \tau_{c}=$ 0.8826 , corresponding to $\tau=0.1$ for the simulation data. Coexisting densities have been joined with tie lines. There is a gap in the compressibility equation curve where the expression for the pressure has no real solution. Scaling the temperatures according to the associated critical points places the simulation results for the low-density branch pressure and the coexisting densities between the two routes of Percus-Yevick theory. We note, however, that the Percus-Yevick pressure is rather unrealistic in the high-density branch, especially from the energy equation, since the pressure must rise very steeply at sufficiently high density.

\section{CONCLUDING REMARKS}

It is noteworthy that, despite there being at least two analytical equations of state for the adhesive hard sphere

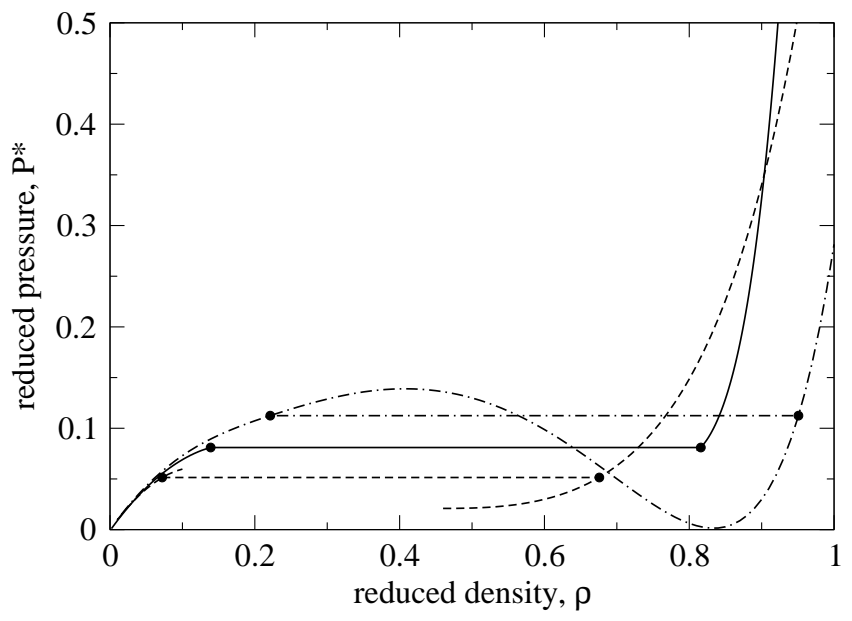

FIG. 6: Adhesive hard sphere pressure on a subcritical isotherm. The solid curve is the simulation result at $\tau=0.1$, equivalent to $\tau / \tau_{c}=0.8826$. The dashed and dot-dashed lines are the Percus-Yevick compressibility and energy results, respectively, each measured at the same $\tau / \tau_{c}$ using the associated $\tau_{c}$. Tie lines join coexisting densities.

model, arising from the compressibility and energy routes of Percus-Yevick theory, it is by far more common for the compressibility results to be cited and used. It is therefore significant that, at least as far as the phase coexistence curve is concerned, the present results place the energy equation closer to the truth.

In summary, we have presented the first firm numerical results for the fluid-fluid coexistence curve of the adhesive hard sphere model, as well as accurate empirical fits to the percolation threshold and the supercritical equation of state. Given the popularity of Baxter's model in the interpretation of experimental data in colloid science, we hope that this information will enhance the model's utility. The results also constitute a reference for future simulations of short ranged attractive systems.

\section{Acknowledgments}

The authors gratefully acknowledge insightful discussions and correspondence with Prof. W. C. K. Poon and Prof. G. Stell. The work of the FOM Institute is part of the research program of FOM and is made possible by financial support from the Netherlands organization for Scientific Research (NWO).

\section{APPENDIX A: MULTIPLE HISTOGRAM}

Recall Eq. (22) for the probability of observing a system of adhesive hard spheres with $N$ particles and $M$ contacts under specified conditions of effective temperature $\tau$ and activity $z$. The continuous probability distribution of the density, $P(\rho ; \tau, z)$ can be approximated 
from the discrete probability in Eq. (22) by summing over $M$ and normalizing with respect to integration over $\rho=\sigma^{3} N / V$ :

$$
P(\rho ; \tau, z)=\frac{\sigma^{3}}{V} \sum_{M} P_{N M}(\tau, z) .
$$

A full knowledge of the density of states $\Omega_{N M}$ to within a multiplicative constant would therefore permit calculation of the density distribution at any combination of temperature and activity using Eqs. (22) and (A1).

In this appendix, we detail our implementation of the multiple histogram technique ${ }^{18,19}$ for adhesive hard spheres. The aim is to combine the data from several simulations at different temperatures and activities to produce an optimal estimate for the density of states over a wide range of $N$ and $M$ so that Eqs. (22) and (A1) can be applied. We follow closely the approach described by Weerasinghe and $\mathrm{Amar}^{38}$ for the canonical ensemble. Our specific adaptation for the adhesive hard sphere model and the grand canonical ensemble is included here for completeness.

Consider a grand canonical simulation at temperature $\tau_{i}$ and activity $z_{i}$, where the subscript $i$ labels the simulation. One can accumulate a two-dimensional histogram of $N$ and $M$. Let $S_{i N M}$ be the number of sampled configurations that had $N$ particles and $M$ contacts, and let $s_{i}=\sum_{N, M} S_{i N M}$ be the total number of samples. Abbreviating $\Xi\left(\tau_{i}, z_{i}\right)$ to $\Xi_{i}$, the histogram furnishes the estimate

$$
S_{i N M} / s_{i} \approx P_{N M}\left(\tau_{i}, z_{i}\right)=\Xi_{i}^{-1} \Omega_{N M} z_{i}^{N} \tau_{i}^{-M},
$$

where the equality is approximate because of the statistical uncertainty inherent in the simulation. If $n_{\text {bins }}$ combinations of $N$ and $M$ are sampled, the corresponding $n_{\text {bins }}$ values of $\Omega_{N M} / \Xi_{i}$ can be obtained from Eq. A2 by straightforward division. The points lying in the tails of the distribution suffer the largest uncertainty due to poor sampling.

If $n_{\text {sim }}$ simulations are performed at different conditions $\left(\tau_{i}, z_{i}\right), 1 \leq i \leq n_{\text {sim }}$, producing $n_{\text {sim }}$ overlapping two-dimensional histograms, we obtain $n_{\text {bins }} \times n_{\text {sim }}$ instances of Eq. (A2). The task of the multiple histogram technique is therefore to find the $n_{\text {sim }}$ values of $\Xi_{i}$ and the $n_{\text {bins }}$ values of $\Omega_{N M}$ that minimize the overall discrepancy between the probabilities they define through Eq. (22) and those measured in the various simulations. By treating these $n_{\text {sim }}+n_{\text {bins }}$ unknowns as individually adjustable parameters, we will obtain the density of states and partition function without making any assumptions about their functional forms.

We start by taking the logarithm of Eq. (A2):

$$
\ln S_{i N M}-\ln s_{i} \approx \ln \Omega_{N M}+N \ln z_{i}-M \ln \tau_{i}-\ln \Xi_{i} .
$$

The left- and right-hand sides of Eq. (A3) are the measured and fitted values of $\ln P_{N M}$, respectively. The discrepancy is the residual,

$$
R_{i N M}=\ln p_{i N M}-\left(\ln \Omega_{N M}+X_{i N M}-\ln \Xi_{i}\right),
$$

where we have introduced the abbreviations $p_{i N M}=$ $S_{i N M} / s_{i}$ and $X_{i N M}=N \ln z_{i}-M \ln \tau_{i}$.

If we assume that sampled configurations are uncorrelated, the variance of $S_{i N M}$ measured in a simulation of finite length is expected to be ${ }^{19} \sigma^{2}\left(S_{i N M}\right)=\left\langle S_{i N M}\right\rangle$, where the angle brackets indicate the true ensemble average over simulations of the same length. If we approximate this average by the value actually measured, then we can estimate the standard deviation of $S_{i N M}$ to be $\sigma\left(S_{i N M}\right)=S_{i N M}^{1 / 2}$. Propagating errors, we find $\sigma\left(\ln p_{i N M}\right)=S_{i N M}^{-1 / 2}$. Summing the squares of the residuals divided by their standard deviations, we arrive at the maximum likelihood estimator

$$
\chi^{2}=\sum_{i} \sum_{N, M} S_{i N M} R_{i N M}^{2}
$$

The first sum in Eq. A5 runs over the $n_{\text {sim }}$ simulations, and the second runs over all bins in the two-dimensional histogram.

We now treat the $n_{\text {sim }}$ values of $\Xi_{i}$ and the $n_{\text {bins }}$ values of $\Omega_{N M}$ as adjustable parameters to be fitted by minimizing $\chi^{2}$. Differentiating Eq. A5 with respect to the fitting variables and equating to zero yields

$$
\begin{aligned}
\partial \chi^{2} / \partial \ln \Omega_{N M} & =-2 \sum_{i} S_{i N M} R_{i N M}=0 \\
\partial \chi^{2} / \partial \ln \Xi_{i} & =2 \sum_{N, M} S_{i N M} R_{i N M}=0
\end{aligned}
$$

Substituting Eq. A4 into Eq. A6 and solving for $\ln \Omega_{N M}$, we obtain $n_{\text {bins }}$ equations of the form

$$
\ln \Omega_{N M}=\sum_{i} q_{i N M}\left(\ln p_{i N M}-X_{i N M}+\ln \Xi_{i}\right)
$$

where $q_{i N M}=S_{i N M} / \sum_{j} S_{j N M}$. Similarly, substituting Eq. (A4) into Eq. (A7) and solving for $\ln \Xi_{i}$, we obtain $n_{\text {sim }}$ equations of the form

$$
\ln \Xi_{i}=\sum_{N, M} p_{i N M}\left(\ln \Omega_{N M}-\ln p_{i N M}+X_{i N M}\right) .
$$

We now have expressions for the partition functions exclusively in terms of the density of states and vice versa. Since there are fewer of the former than the latter, we solve for the partition functions first. Substituting Eq. (A8) into Eq. (A9) and yields

$$
\begin{aligned}
\ln \Xi_{i} & =\sum_{N, M} p_{i N M}\left[-\ln p_{i N M}+X_{i N M}\right. \\
& \left.+\sum_{j} q_{j N M}\left(\ln p_{j N M}-X_{j N M}+\ln \Xi_{j}\right)\right] .
\end{aligned}
$$


Gathering terms in $\Xi_{i}$ on the left-hand side gives

$$
\begin{aligned}
\ln \Xi_{i} & -\sum_{N, M} p_{i N M} \sum_{j} q_{j N M} \ln \Xi_{j} \\
& =\sum_{N, M} p_{i N M} \sum_{j}\left(q_{j N M}-\delta_{i j}\right)\left(\ln p_{j N M}-X_{j N M}\right),
\end{aligned}
$$

where $\delta_{i j}$ is the Kronecker delta.

Eq. (A11) is a set of $n_{\text {sim }}$ linear equations in $n_{\text {sim }}$ unknowns, and can be written in matrix notation as

$$
\mathbf{A Y}=\mathbf{B}
$$

where $Y_{i}=\ln \Xi_{i}$. The elements of the matrix $\mathbf{A}$ are

$$
A_{i j}=\delta_{i j}-\sum_{N, M} p_{i N M} q_{j N M}
$$

and the elements of the vector $\mathbf{B}$ are

$$
B_{i}=\sum_{N, M} p_{i N M} \sum_{j}\left(q_{j N M}-\delta_{i j}\right)\left(\ln p_{j N M}-X_{j N M}\right) .
$$

1 R. J. Baxter, J. Chem. Phys. 49, 2770 (1968).

2 R. O. Watts, D. Henderson, and R. J. Baxter, Adv. Chem. Phys. 21, 421 (1971).

3 Y. C. Chiew and E. D. Glandt, J. Phys. A 16, 2599 (1983).

4 S. V. G. Menon, V. K. Kelkar, and C. Manohar, Phys. Rev. A 43, 1130 (1991).

5 S. H. Chen, J. Rouch, F. Sciortino, and P. Tartaglia, J. Phys. Cond. Mat. 6, 10855 (1994).

${ }^{6}$ K. Dawson, G. Foffi, W. Götze, F. Sciortino, M. Sperl, P. Tartaglia, T. Voigtmann, and E. Zaccarelli, Phys. Rev. E 63, 011401 (2001).

7 K. N. Pham, A. M. Puertas, J. Bergenholtz, S. U. Egelhaaf, A. Moussaïd, P. N. Pusey, A. B. Schofield, M. E. Cates, M. Fuchs, and W. C. K. Poon, Science 296, 104 (2002).

8 N. A. Seaton and E. D. Glandt, J. Chem. Phys. 86, 4668 (1987).

9 N. A. Seaton and E. D. Glandt, J. Chem. Phys. 87, 1785 (1987).

10 W. G. T. Kranendonk and D. Frenkel, Mol. Phys. 64, 403 (1988).

11 S. B. Lee, J. Chem. Phys. 114, 2304 (2001).

12 G. Stell, J. Stat. Phys. 63, 1203 (1991).

13 G. Ciccotti and J. P. Ryckaert, Comp. Phys. Rep. 4, 346 (1986).

14 N. A. Seaton and E. D. Glandt, J. Chem. Phys. 84, 4595 (1986).

15 S. B. Lee, J. Chem. Phys. 98, 8119 (1993).

16 D. J. Adams, Mol. Phys. 28, 1241 (1974).

17 C. J. Geyer and E. A. Thompson, J. Am. Stat. Assoc. 90, 909 (1995).

18 A. M. Ferrenberg and R. H. Swendsen, Phys. Rev. Lett. 61, 2635 (1988).

19 A. M. Ferrenberg and R. H. Swendsen, Phys. Rev. Lett.
One practical point in evaluating $\mathbf{A}$ and $\mathbf{B}$ is that many of the $S_{i N M}$ are zero, making $\ln p_{i N M}$ and $\ln q_{i N M}$ incalculable. However, such logarithms always appear in products involving $S_{i N M}$ itself. Since $\lim _{x \rightarrow 0} x \ln x=0$, bins with zero contents simply make no contribution to Eqs. (A5), (A13) and (A14).

Solution of Eq. A12 leads to numerical values for the partition functions, which can then be substituted into Eq. (A8) to obtain the density of states. In principle, however, one cannot obtain the absolute values of the partition functions from a set of equations like (22), only their ratios. For this reason, the matrix $\mathbf{A}$ is singular, and Eq. A12 should be tackled using singular-value decomposition, ${ }^{35}$ yielding $\Xi_{i}$ and $\Omega_{N M}$ up to a multiplicative constant.

63, 1195 (1989).

20 B. Borštnik, C. G. Jesudason, and G. Stell, J. Chem. Phys. 106, 9762 (1997).

21 M. H. J. Hagen, E. J. Meijer, G. C. A. M. Mooij, D. Frenkel, and H. N. W. Lekkerkerker, Nature (London) 365, 425 (1993).

22 M. A. Miller and D. Frenkel, Phys. Rev. Lett. 90, 135702 (2003).

23 M. M. Tsypin and H. W. J. Blöte, Phys. Rev. E 62, 73 (2000).

24 A. D. Bruce and N. B. Wilding, Phys. Rev. Lett. 68, 193 (1992).

25 N. B. Wilding and A. D. Bruce, J. Phys. Cond. Mat. 4, 3087 (1992).

26 N. B. Wilding, Phys. Rev. E 52, 602 (1995).

27 R. Piazza, V. Peyre, and V. Degiorgio, Phys. Rev. E 58, R2733 (1998).

28 F. Mallamace, P. Gambadauro, N. Micali, P. Tartaglia, C. Liao, and S.-H. Chen, Phys. Rev. Lett. 84, 5431 (2000).

29 D. Frenkel and B. Smit, Understanding Molecular Simulation (Academic Press, San Diego, 2002), 2nd ed.

${ }^{30}$ W. C. K. Poon and M. D. Haw, Adv. Colloid Interface Sci. 73, 71 (1997).

31 R. J. Speedy, J. Phys. Cond. Mat. 9, 8591 (1997).

32 A. J. Post and E. D. Glandt, J. Chem. Phys. 84, 4585 (1986)

33 J. J. Nicolas, K. E. Gubbins, W. B. Streett, and D. J. Tildesley, Mol. Phys. 37, 1429 (1979).

34 J. K. Johnson, J. A. Zollweg, and K. E. Gubbins, Mol. Phys. 78, 591 (1993).

35 W. H. Press, S. A. Teukolsky, W. T. Vetterling, and B. P. Flannery, Numerical Recipes in FORTRAN (Cambridge University Press, Cambridge, 1992), 2nd ed. 
36 B. Barboy, J. Chem. Phys. 61, 3194 (1974).

(1993)

37 B. Barboy and R. Tenne, Chem. Phys. 38, 369 (1979).

38 S. Weerasinghe and F. G. Amar, J. Chem. Phys. 98, 4967 